\title{
Spatial/Spectral Resolution of a Galactic Bulge K3 Giant Stellar Atmosphere via Gravitational Microlensing
}

\author{
Sandra Castro ${ }^{1}$, Richard W. Pogge ${ }^{2}$, R. Michael Rich ${ }^{3}$, \\ D. L. DePoy ${ }^{2}$, Andrew Gould ${ }^{2,4}$
}

\begin{abstract}
We present two Keck HIRES spectra $(\lambda / \Delta \lambda=40,000)$ of a bulge K3 giant taken on successive nights during the second caustic crossing of the binary microlensing event EROS BLG-2000-5. This caustic crossing served effectively to resolve the surface of the source star: the spectrum from the second night is dominated by the limb, while the spectrum from the first night is comprised of light from a broader range of radii. To demonstrate that the spectra are adequate to resolve the differences between them, we analyze the $\mathrm{H} \alpha$ line. The equivalent width is $\sim 8 \%$ smaller on the second night, and the signal-to-noise ratio per resolution element (165 and 75 on the two nights respectively) is sufficient to show that the difference is approximately constant over the $\sim 2 \AA$ $(\sim 15$ resolution element) extent of the line. The sign of the difference is in the expected direction since the limb is the coolest part of the star and therefore should have the weakest $\mathrm{H} \alpha$. We invite atmosphere modelers to predict the difference spectrum from the entire spectral range $5500<\lambda<7900 \AA$ so that these predictions can be compared to our observations.
\end{abstract}

Subject headings: gravitational lensing - stars: atmospheres - stars: imaging stars: individual (EROS BLG-2000-5)

\footnotetext{
${ }^{1}$ Department of Astronomy, Caltech, Pasadena, CA, 91125. email: smc@astro.caltech.edu

${ }^{2}$ Department of Astronomy, The Ohio State University, 140 W. 18th Ave, Columbus, OH 43210-1173. email: pogge, depoy, gould@astronomy.ohio-state.edu

${ }^{3}$ Department of Physics \& Astronomy, University of California, Los Angeles, CA, $90095 . \quad$ email: rmr@astro.ucla.edu

${ }^{4}$ Laboratoire de Physique Corpusculaire et Cosmologie, College de France, 11 pl. Marcelin Berthelot, F-75231, Paris, France
} 


\section{Introduction}

The spectrum of the Sun varies as a function of position on the Sun's disk. The surface of last scattering lies deeper in the atmosphere at the center than it does at the limb. The center therefore appears hotter, and therefore brighter and bluer. Spectral lines measured near the center (compared to those at the limb) reflect the hotter conditions at the surface of last scattering and also bear the imprint of a different pattern of absorption features imposed upon the spectrum as the light traverses a different path through the atmosphere.

These same effects are presumably present in all stars and are routinely taken into account when modeling stellar atmospheres. However, they have never been directly observed in any star except the Sun and nearby supergiants like Betelgeuse (e.g., Uitenbroek, Dupree \& Gilliland 1998), because the angular sizes of other stars are generally too small to take spectra at different spatial positions. Limb darkening coefficients have also been derived for a handful of eclipsing binary stars from a combination of spectroscopy and light curves (e.g., GZ Cma; Popper et al. 1985). Hence, while spatially resolved models of stellar atmospheres are well tested for solar type stars, they are poorly tested for most other types of stars, in particular giants and metal-poor stars. For these, models and observations can be compared only for the integrated spectra.

Microlensing can be used to measure limb darkening. Microlensing magnification patterns have caustics, (infinitesimally small) loci of formally infinite magnification. If the source passes over a caustic, then at different times different parts of the source are highly magnified, so from a time series of flux measurements one can reconstruct the stellar brightness profile.

When Witt (1995) first proposed this idea, he specifically considered microlensing by a point lens. In fact, all four limb-darkening measurements made to date (Albrow et al. 1999, 2000, 2001; Afonso et al. 2000) used binary microlensing events.] This is no accident. Point lenses have point-like caustics, so the cross section for a caustic crossing is the angular diameter of the source. By contrast, binary lenses have one to three closed linear caustics with total cross sections that tend to be more than two orders of magnitude larger than the source. Thus, even though recognizable binary microlensing events are relatively rare, they comprise the great majority of caustic crossing events.

Valls-Gabaud $(1996,1998)$ showed that stellar atmospheres could be resolved spectrally by applying the same principle to a time series of spectra rather than broad-band

\footnotetext{
${ }^{5}$ Alcock et al. (1997) marginally detected limb darkening in a point-lens event, but could not measure a limb-darkening coefficient.
} 
measurements. Heyrovský, Sasselov, \& Loeb (2000) made detailed predictions of how such spectra might vary during a microlensing event. Several other closely related applications of time series of microlensing spectra were presented by Maoz \& Gould (1994), Gould (1997), and Ignace \& Hendry (1999).

All of these theoretical investigations focused on point lenses. Gaudi \& Gould (1999) argued that to be practical, spatial/spectral resolution would require binary-lens caustics. The most important reason is that binary caustic crossings can be accurately predicted in advance: binary caustics are closed curves, so once the caustic region is entered, there must inevitably be an exit crossing. The approach to this crossing is a square-root singularity which can be modeled without detailed knowledge of the (generally complex) full binary-lens light curve. Gaudi \& Gould (1999) made a detailed study of the signal-to-noise ratio $(\mathrm{S} / \mathrm{N})$ characteristics of such observations. Their most important conclusion in this regard was that observations during the second half of the crossing (between the times when the center and the trailing limb exits the caustic) carry substantially more spatial-resolution information than those during the first half (see their Figure 4b).

The fundamental reason for this can be understood from Figure @. When the source is inside a caustic, it has five images, so the magnification can be written $A=A_{2}+A_{3}$, where $A_{2}$ is the magnification of the two images that brighten divergently (and then disappear) as the source approaches the caustic, and $A_{3}$ is the magnification of the three other images. To a good approximation, $A_{3}$ can be regarded as a constant during the crossing, and

$$
A_{2}=\left(\frac{\rho_{*}}{u_{r}}\right)^{-1 / 2}\left(-\frac{\theta_{\perp}}{\theta_{*}}\right)^{-1 / 2} \Theta\left(-\theta_{\perp}\right) .
$$

Here $\theta_{\perp}$ is the angular separation of a given position in the source plane from the caustic, $\theta_{*}$ is the angular source size, $\rho_{*}=\theta_{*} / \theta_{\mathrm{E}}, \theta_{\mathrm{E}}$ is the angular Einstein radius, $u_{r}$ is a parameter that depends on the details of the lens geometry, and $\Theta$ is a step function. Typically $u_{r} \sim 1$, $\rho_{*} \lesssim 10^{-2}$, and $A_{3}$ is of order a few. Hence, during a caustic crossing, the magnification is dominated by $A_{2}$. Figure 1 shows the relative magnification due to $A_{2}$ of different concentric rings on the stellar surface during different phases of the crossing. These phases are characterized by $\eta$, the angular distance from the center of the source to the caustic in units of $\theta_{*}$. Note that for the two curves with $\eta>0$ (second half of the crossing), the $A_{2}$ images sample roughly uniformly the outer part of the $\operatorname{star}\left(\theta / \theta_{*}>\eta\right)$, but do not sample the inner part at all. By taking a series of such spectra, one can deconvolve the spatial profile. By contrast, while the $\eta<0$ curves possess a sharp feature near $\theta / \theta_{*}=-\eta$, its effect is muted by the high and roughly uniform magnification from the rest of the star. Here $\theta / \theta_{*}$ is the fractional distance of a given concentric ring from the center of the star. Note that this formalism cannot be applied to cusp-crossing events or more generally to events 
where some parts of the source are roughly equidistant from two caustics while other parts are crossing a caustic. These constitute a significant fraction of observed caustic-crossing events (e.g., Alcock et al. 2000) and must be handled differently (e.g., Albrow et al. 1999).

Alcock et al. (1997) acquired spectra of the high-magnification event MACHO 95-BLG-30 and observed changes in $\mathrm{H} \alpha$ and $\mathrm{TiO}$ near $6700 \AA$ suggestive of center-to-limb variations in the spectral lines. Lennon et al. (1996) attempted to observe spectroscopic changes during the second caustic crossing of the binary microlensing event MACHO 96-BLG-3 using the ESO NTT spectrograph, but failed to see any. They concluded that 8-10 m class telescopes would be required.

In this Letter we present high-resolution spectroscopy of a caustic-crossing microlensing event of a K3 giant. We demonstrate that we have indeed resolved the stellar atmosphere by comparing the $\mathrm{H} \alpha$ absorption lines in spectra taken on successive nights during the second half of the crossing. We show that $\mathrm{H} \alpha$ absorption is significantly smaller in equivalent width on the second night when the magnified light is dominated by the limb. This is qualitatively expected, since the limb is cooler than the center, and therefore effectively corresponds to a later spectral type that has weaker hydrogen lines.

The spectra contain a vast wealth of information. To extract the quantitative implications for the atmosphere requires a comparison of the data with the predictions from detailed atmospheric models convolved with the precise magnification profiles at the times of the observations as determined from detailed modeling of the photometric light curves, neither of which are available at present. However, we invite modelers to make their predictions so that they may be compared with the observations in a future paper.

\section{Observations}

The EROS collaboration issued an alert on 2000 April 29 that EROS BLG-2000-5 was a probable microlensing event with a clump giant source toward the Galactic bulge (www-dapnia.cea.fr/Spp/Experiences/EROS/alertes.html). On 2000 June 8, the MPS collaboration (bustard.phys.nd.edu/MPS/index.html) issued an anomaly alert, noting rapid source brightening. The PLANET collaboration (thales.astro.rug.nl/ planet) then began intensive observations within 4 hours of the onset of the first crossing, measuring its duration to be $2 \Delta t_{1}=1.0$ days.

PLANET issued further anomaly alerts, identifying the source as a K3 giant and predicting the date of the second crossing. By real-time modeling of their observations, PLANET predicted that the source would cross the second caustic at a much more acute 
angle than the first, and therefore the second crossing would last several days. [

This extremely long crossing set the stage for Keck observations which otherwise would probably not have been feasible. Normally, the duration of the second-half crossing (which Gaudi \& Gould 1999 showed contains most of the information) is only a few hours. The chance that it will be visible from a given observatory site is therefore small, and the chance that it will be visible over a wide range of $\eta$ (needed to obtain differential measurements of the stellar atmosphere) is smaller still. However, a crossing that lasts several days can be seen at substantially different $\eta$ simply by viewing it on different nights. In their last alert prior to the onset of the observations described below, PLANET predicted that the center of the source would cross the second caustic on HJD=2451731.6, and predicted that the duration of the second-half crossing would be about 2 days. They urged immediate spectroscopic observations, which we then undertook.

\subsection{Spectroscopic Observations}

We obtained spectra of the bulge K3 giant star being lensed by EROS BLG-2000-5 on UTC 2000 July 6 and 7 using the High Resolution Spectrograph (HIRES; Vogt 1994) with the W. M. Keck 10-m telescope. The spectra cover the range $5500<\lambda<7900 \AA$ at resolution $\lambda / \Delta \lambda \approx 40,000$. On July 6 we took three exposures of 1800s starting at 09:57:26 UTC, and on July 7 we took four 1800s exposures starting at 09:35:48 UTC. Total integration times were $5400 \mathrm{~s}$ and $7200 \mathrm{~s}$ on the respective nights. The mid-exposure Heliocentric Julian dates of the combined spectra are HJD $=2451731.953$ and HJD=2451732.950, respectively. The spectra of July 6 were taken in 0 ".8 seeing through light cirrus using the C1 slit decker. This gave slit dimensions of $0^{\prime \prime} 86 \times 7^{\prime \prime} .0$, providing a resolution of $\sim 45,000$. On the second night the seeing was 1 1". $2-1$ ". 4 through heavy variable cirrus clouds. To improve the $\mathrm{S} / \mathrm{N}$ of the spectra we used the C5 slit decker which has slit dimensions of $1^{\prime \prime} .15 \times 7^{\prime \prime}$. 0 , giving a resolution of $\sim 34,000$. The $\mathrm{S} / \mathrm{N}$ ratios in the $\mathrm{H} \alpha$ echelle order are $\sim 90$ and $\sim 40$ per pixel (165 and 75 per resolution element) for July 6 and 7, respectively. The lower $\mathrm{S} / \mathrm{N}$ ratio on the second night spectrum reflects the poorer photometric conditions, and does not affect the $\mathrm{H} \alpha$ line analysis.

The data were reduced using the Mauna Kea Echelle Extraction - MAKEE (T. Barlow)

\footnotetext{
${ }^{6}$ For sufficiently acute crossings, especially near a cusp, the approximation of constant $u_{r}$ (embodied in eq. [1]) can break down. This has no effect on the arguments and results presented here, but it must be taken into account in detailed modeling of the event. For EROS BLG-2000-5 the approximation is likely to remain valid.
} 
program for HIRES. It is optimized for the spectral extraction of single, unresolved point sources. Data reduction was done independently in a variety of ways in order to check for systematics in the procedure. The continuum was fit individually to each echelle order (covering $\sim 100 \AA$ ) using a hot star observed during the same night and 5th order polynomials. The K3 giant spectra in each order were then divided by the continuum fits to normalize the spectra.

We tested for and excluded such potentially confusing systematic effects as faint nearby stars, sky background, and scattered light. Contamination from the lens itself cannot be eliminated since they are separated by $\sim 1$ mas. However, this contamination is expected to be small since the source is both intrinsically bright and highly magnified.

\section{Results}

Figure 2 shows the portion of the spectra centered on the $\mathrm{H} \alpha$ absorption line from the two nights. The spectra were first each normalized to a mean continuum of unity. The spectra were aligned in wavelength by removing a small residual shift of 0.256 pixels from the July 7 spectrum, and the resolutions were matched by convolving the July 6 spectrum with a 2.46 pixel FWHM Gaussian. The wavelength scale is $47 \mathrm{~m} \AA /$ pixel. The upper panel shows the spectra themselves, with key lines identified, while the lower panel shows the fractional change in line flux with wavelength, $\delta F_{\lambda}=2\left(F_{\lambda, 6}-F_{\lambda, 7}\right) /\left(F_{\lambda, 6}+F_{\lambda, 7}\right)$, where $F_{\lambda, 6}$ and $F_{\lambda, 7}$ are the normalized fluxes (shown in the upper panel) for July 6 and July 7 respectively.

Figure 3 provides an expanded view of Figure 2 for the $\mathrm{H} \alpha$ line alone. The fractional change in the line fluxes as a function of wavelength is reasonably consistent with a constant value, $\sim 0.1$. The equivalent widths of the lines are $944 \pm 2 \mathrm{~m} \AA$ and $869 \pm 6 \mathrm{~m} \AA$ on July 6 and 7 , respectively, measured from the nominal continuum level in each spectrum. These equivalent widths differ by $8.3 \% \pm 0.7 \%$.

Since the two spectra were taken a day apart and the second-half crossing time was predicted by PLANET to be about 2 days, the source had changed its separation from the caustic by $\Delta \eta \sim 0.5$ in the interval between the observations. Hence, these spectra correspond roughly to the magnification profiles labeled $\eta=0.25$ and $\eta=0.75$ in Figure 1. That is, the July 7 spectrum is concentrated near the limb, while the July 6 spectrum covers a much broader range of stellar radii. Thus, one expects that the July 7 spectrum reflects conditions higher in the atmosphere, implying a cooler effective temperature, and so weaker hydrogen lines. This is exactly what is seen. It also agrees with the predictions of 
Heyrovský et al. (2000), although precise comparison is not possible, in part because they considered point-lens caustics rather than binary-lens caustics, and in part because they were modeling $\mathrm{M}$ stars rather than $\mathrm{K}$ stars.

The behavior of adjacent spectral lines indicates that the difference we see in the $\mathrm{H} \alpha$ line is real and not an artifact of the reduction process or other systematic effects. In particular, the Ca I $\lambda 6572.9 \AA$ line and the Fe I $\lambda 6569.2 \AA$ line, which both lie in the same order as $\mathrm{H} \alpha$ (see Fig.2), show no detectable difference in equivalent width between the two nights $(228.4 \pm 2.3 \mathrm{~m} \AA$ and $228.8 \pm 2.7 \mathrm{~m} \AA$ for $\mathrm{Ca} \mathrm{I}$, and $142.0 \pm 3.0 \mathrm{~m} \AA$ and $149.3 \pm 3.3 \mathrm{~m} \AA$ for Fe I). Not all lines are unchanged, for example the V I+Sc I blend at $6558 \AA(111.4 \pm 2.2 \mathrm{~m} \AA$ compared to $77.8 \pm 4.7 \mathrm{~m} \AA$ ). What is important is that while some differences are seen, we do not see systematic differences among all lines.

\section{Conclusions}

We have presented spectra of a K3 giant taken on two different nights during the second caustic crossing of the microlensing event EROS BLG-2000-5. The spectrum from the second night tends to be dominated by light from the limb of the source, while the spectrum from the first night covers a much wider range of radii. Naively, one therefore expects the second spectrum to reflect cooler conditions and so to display weaker hydrogen lines. The difference spectrum agrees with this qualitative prediction.

To make more quantitative predictions for changes in this line (and in all other spectral features covered by our spectra), two additional inputs will be required: 1) the event caustic geometry, and 2) the limb-darkening profile of the source. Both can be obtained from detailed modeling of the photometric light curve. It is clear from Figure 1 that knowing the geometry, particularly $\eta_{\text {July } 6}$ and $\eta_{\text {July }}$, is critically important. The effect of $A_{3}$ is to put a small uniform pedestal under all curves in Figure 1 and is therefore not as crucial. The effect of limb darkening can be quite significant, as can be seen from the limb darkening of a K2 giant shown in Figure 8 of Albrow et al. (1999).

When these various parameters are measured, we invite atmosphere modelers to combine them with their atmosphere models to predict the difference spectra. It will then be possible to directly compare these predictions with our data.

We thank the EROS, MPS, and PLANET collaborations for providing the microlensing event alerts that made these observations possible. We thank Fred Chaffee, director of the W.M. Keck Observatory, for bringing the alerts to the attention of the observers, and 
Don Terndrup for useful suggestions. Data were obtained at the W.M. Keck Observatory, which is operated as a scientific partnership among the California Institute of Technology, the University of California, and the National Aeronautics and Space Administration. The Observatory was made possible by the generous financial support of the W.M. Keck Foundation. This work was supported by NSF grants AST-9727520 and AST-9530619, NASA grant NAG5-7589, and a grant from Le Ministère de L'Éducation Nationale de la Recherche et de la Technologie.

\section{Note Added in Proof:}

After we received the referee's comments to the original version of this paper, we learned of the work of Albrow et al. (astro-ph/0011380) reporting complementary low-resolution observations of the same caustic crossing. 


\section{REFERENCES}

Afonso, C., et al. 2000, ApJ, 532, 340

Albrow, M.D., et al. 1999, ApJ. 522, 1011

Albrow, M.D., et al. 2000, ApJ, 534, 894

Albrow, M.D., et al. 2001, ApJ, 549, 000 astro-ph/0004243

Alcock, C. et al. 1997, ApJ, 491, 436

Alcock, C. et al. 2000, ApJ, 541, 270

Gaudi, B.S., \& Gould, A. 1999, 513, 619

Gould, A. 1997, 483, 98

Heyrovský, D., Sasselov, D.D., \& Loeb, A. 2000, ApJ, submitted (astro-ph/9902273)

Ignace, R., \& Hendry, M.A. 1999, A\&A, 341, 201

Lennon, D.J., Mao, S., Fuhrmann, K., \& Gehren, T. 1996, ApJ, 471, L23

Maoz, D., \& Gould, A. 1994, ApJ, 425, L67

Popper, D.M., Andersen, J., Clausen, J.V., \& Nordstrom, B. 1985, AJ, 90, 1324

Uitenbroek, H., Dupree, A.K., \& Gillilan, R.L. 1998, AJ, 116, 2501

Valls-Gabaud, D. 1996, in IAU Symp. 173, Astrophysical Applications of Gravitational Lensing, ed. C.S. Kochanek \& J.N. Hewitt (Dordrecht: Kluwer), 237

Valls-Gabaud, D. 1998, MNRAS, 294, 747

Vogt, S., et al. 1994, SPIE, 2198, 362

Witt, H.J. 1995, ApJ, 449, 42 


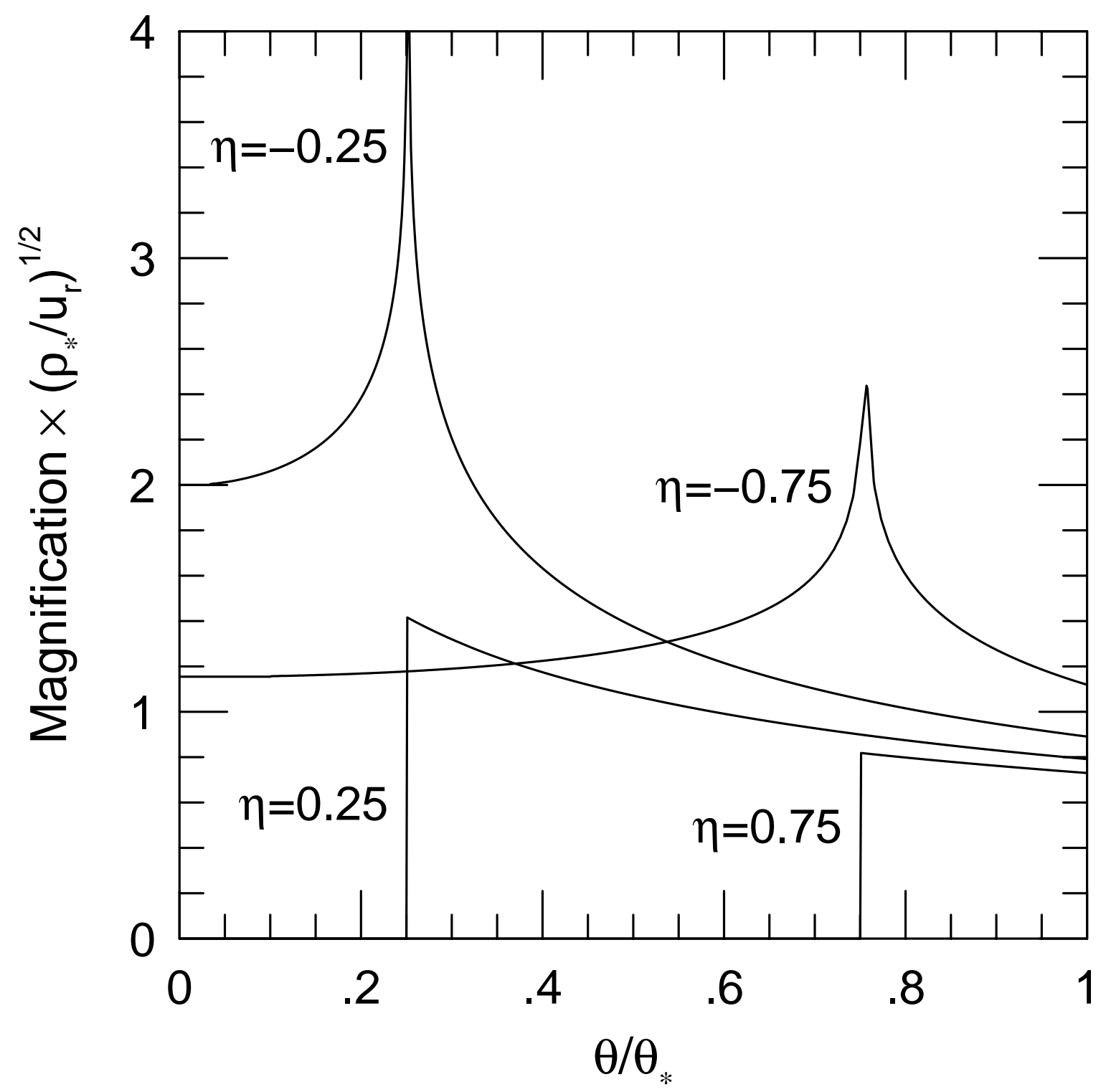

Fig. 1.- Relative magnification averaged over concentric stellar radii, $\theta$, at various phases, $\eta$, due to the two extra images during a binary-lens caustic crossing. Here $\eta$ is the separation between the center of the source and the caustic in units of the source radius, $\theta_{*}$. During the second half of the crossing $(\eta>0)$, there is a sharp cutoff in the magnification for $\theta / \theta_{*}<\eta$. The stellar profile can be deconvolved from a series of spectra taken during this interval. During the first half of the crossing $(\eta<0)$, there are features in the magnification profile at $\theta / \theta_{*}=-\eta$, but these are much less pronounced, so deconvolution would be much more difficult. See also Fig. 4b from Gaudi \& Gould (1999). The magnification profile is proportional to the parameter combination $\left(\rho_{*} / u_{r}\right)^{-1 / 2}$. Typically $10^{-2} \gtrsim \rho_{*} / u_{r} \gtrsim 10^{-3}$, so the magnifications are scaled up from this figure by a factor 10-30. 


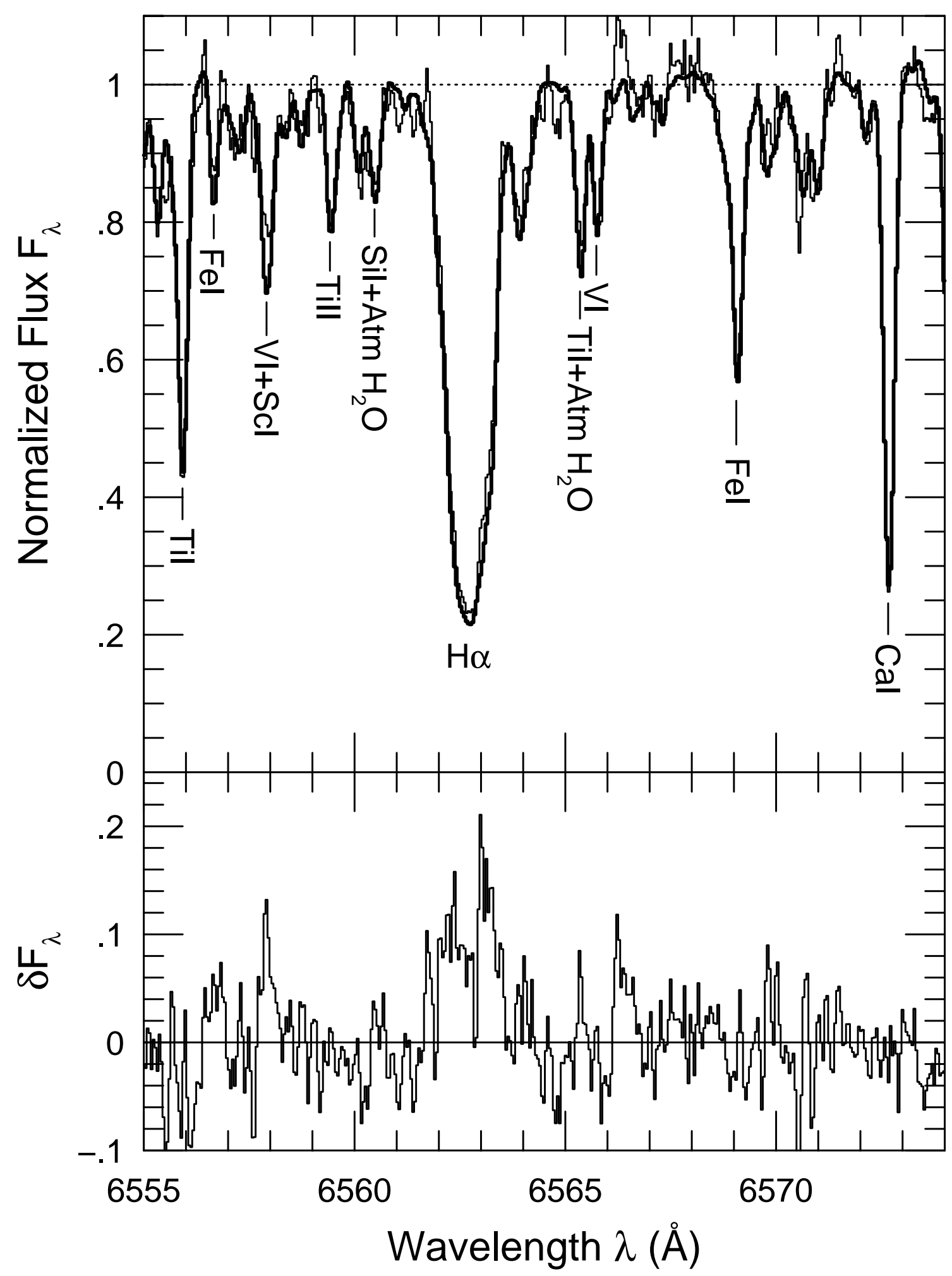

Fig. 2.- Upper panel: HIRES spectra of EROS BLG-2000-5 on UTC 2000 July 6 (bold) and 2000 July 7 (solid) around $\mathrm{H} \alpha$ (one order). Lower Panel: Fractional difference in the lines as a function of wavelength: $\delta F_{\lambda}=2\left(F_{\lambda, 6}-F_{\lambda, 7}\right) /\left(F_{\lambda, 6}+F_{\lambda, 7}\right)$, where $F_{\lambda, 6}$ and $F_{\lambda, 7}$. 


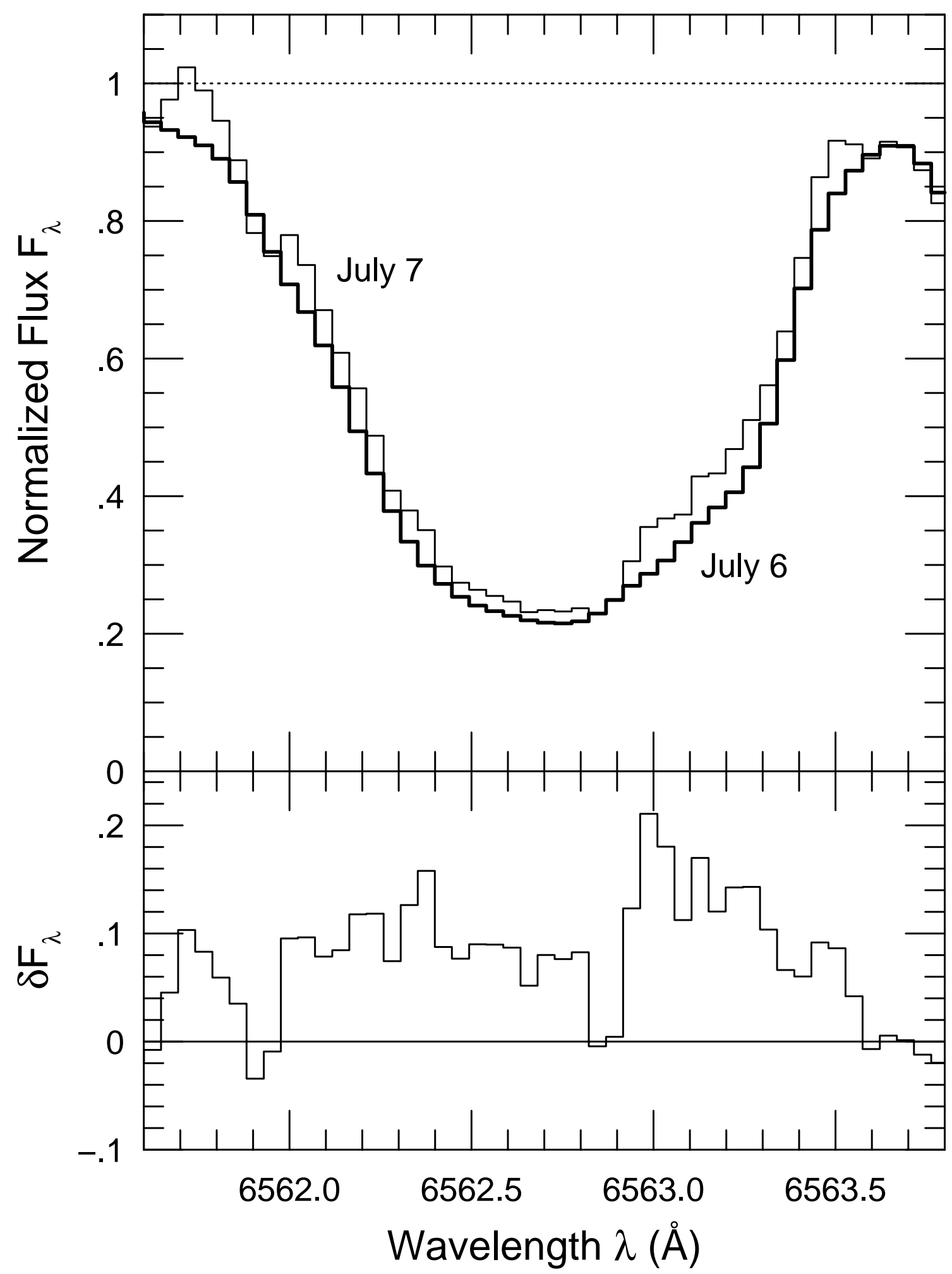

Fig. 3.- Detail of Figure 2 centered on the $\mathrm{H} \alpha$ line. 